\title{
The ESCAPE Dark Matter Test Science Project
}

\author{
Elena Cuoco, ${ }^{a}$ Caterina Doglioni,,${ }^{b, *}$ Kay Graf, ${ }^{c}$ Giovanni Lamanna ${ }^{d}$ and Samuel \\ Meehan $^{e}$ \\ ${ }^{a}$ Lund University, Sweden \\ ${ }^{b}$ EGO and Scuola Normale Superiore di Pisa, Italy \\ ${ }^{c}$ LAPP (Laboratoire d'Annecy de Physique des Particules), CNRS/IN2P3 and USMB, France \\ ${ }^{d}$ FAU, Germany \\ ${ }^{d}$ CERN, Switzerland \\ E-mail: caterina.doglioni@hep.lu.se
}

\begin{abstract}
A Dark Matter Science Project is being developed in the context of the ESCAPE project. The goal of this ESCAPE Science Project is to highlight the synergies between different communities and experiments searching for dark matter by producing new results and making the necessary data and software tools fully available, in particular focusing on data management, data analysis and computing. As part of this Science Project, we will use experimental data and software procedures from selected direct detection, indirect detection, and particle collider experiments involved in ESCAPE as prototypes for sustainable end-to-end analysis pipelines.
\end{abstract}

Tools for High Energy Physics and Cosmology - TOOLS2020

2-6 November, 2020

Institut de Physique des 2 Infinis (IP2I), Lyon, France

\footnotetext{
${ }^{*}$ Speaker
} 
One overarching objective of science is to further our understanding of the universe and its composition. The nature of dark matter, corresponding to $85 \%$ of the matter currently present in the universe is still unknown [1]. The presence and distribution of dark matter is detected through its gravitational interactions by observatories and experiments, while the interactions of dark matter with ordinary matter particles can be observed indirectly and directly in astrophysics experiments [2,3]. These interactions also allow for dark matter to be produced in collisions of ordinary matter and observed in experiments at colliders [4] and at particle accelerators [5], and provide complementary information about dark matter - ordinary matter interactions. Data from this wealth of astrophysics and particle physics experiments, combined with theoretical models and interpretations, will shed new light on dark matter.

The presence of dark matter in astrophysical observations, combined with the absence of clues for dark matter particles in experiments, indicates that if dark matter has interactions with ordinary matter they must be very feeble, and produce subtle experimental signals. Connecting results and potential discoveries from different experiments requires the involvement of all communities involved - astrophysics, particle physics and nuclear physics, as recommended within the update of the European Strategy of Particle Physics [6, 7].

Besides the interpretation of results in terms of dark matter theories, synergies also exist between different communities and experiments in terms of tools needed to produce those results, in particular in terms of data management, data analysis and computing. This is why this LOI presents the Dark Matter Science Project within the European Science Cluster of Astronomy and Particle physics ESFRI research infrastructures https://projectescape.eu, to create a link between dark matter as a fundamental science question and the computational tools needed to answer it.

The scientific added value for this TSP is provided by new dark matter analyses coming from the complementary experiments involved (particle colliders, direct and indirection experiments) as well as from theory and observational constraints, all interpreted within the same theoretical framework and displayed in a summary plot that showcases their synergies. During the analysis design, we will identify innovative algorithms (e.g. machine learning, but also procedures to reconstruct images to distinguish signal and background) that can be individually highlighted and shared for use by other scientific communities and / or in society. The Open Science added value for this TSP is that all the digital objects within these new DM analyses will be implemented within the ESCAPE services infrastructure. We will make use of the ESCAPE Data Infrastructure for Open Science in the European Open Science Cloud to store, distribute and provide data and software access to the broad dark matter scientific community. This is a unique link between DM as a fundamental science question and the Open Science services needed to answer it that benefits the scientific community as a whole.

As part of this Science Project, we will convert the existing experimental data and software procedures to sustainable analysis pipelines as a prototype for selected direct detection, indirect detection, and collider experiments involved in ESCAPE, relying on the ESCAPE service infrastructure. We will make use of the ESCAPE Data Infrastructure for Open Science in the European Open Science Cloud to store, distribute and provide data access to the dark matter scientific community, also making this data searchable, while respecting experiment Open Data Policies. The work within this Science Project aims to include all the science data and digital objects (for example data management, metadata, reconstruction software, analysis pipelines, simulations...). The final 
output of each workflow will be individual experimental curves to be interpreted in terms of dark matter particle properties, that can then be combined or compared within summary plots for certain dark matter models. The pipelines will also be designed so that they can ultimately automatically (re)produce this kind of plots with new models.

A related objective of this Science Project is to support the creation of a collection of versioned repositories for experimental results as well as for theory predictions and interpretations. We will implement this service by making available experimental data, results and their interpretations discoverable through the ESCAPE Science Analysis Platform.

A further objective of this Science Project is to catalogue and create new resources for citizen science and crowdsourced data mining, aided by citizen participation within the ESCAPE Citizen Science program. Finally, the dark matter-Test Science Project will help dark matter researchers with the implementation of Particle Physics Masterclass exercises in which high school students can become dark matter scientists for a day and analyse experimental data.

As part of this Science Project, we will hold "hackathons" in collaboration with the HEP Software Foundation [8] to both educate a broader community about existing tools available for analysis preservation (e.g. REANA) and to further apply these tools to more dark matter analyses. These hackatons will produce further examples of sustainable end-to-end science workflows for dark matter experiments.

With this Science Project, we will contribute to the development of the European Open Science Cloud using the ESCAPE tools as a mean to store, distribute and provide FAIR data access to the dark matter scientific community within the experimental Open Data Policies.

In summary, this Science Project aims to further understand the nature of DM by performing new analyses within the experiments involved, and collecting all the digital objects related to those analyses (data, metadata and software) on a broad platform that will be ultimately hosted on the EOSC Portal and will allow these analyses to be reproducible within the various collaboration and by the entire community wherever possible. It will exploit synergies across different communities, as the final output of each workflow will be individual experimental curves that can be interpreted in terms of dark matter particle properties and displayed on the same plots summarizing results from complementary experiments. Furthermore, this Science Project will enhance the research participation in the European Open Science Cloud, by providing a working example of open science that has started from a bottom-up effort by different experiments.

Note: The Dark Matter Science Project will kick-off in 2021, and once the work is underway we will contact the TOOLS2020 participants who have expressed interest to be informed of the evolution of the project.

\section{References}

[1] G. Bertone and D. Hooper, History of dark matter, Rev. Mod. Phys. 90 (2018) 045002 [1605.04909].

[2] J. M. Gaskins, A review of indirect searches for particle dark matter, Contemp. Phys. 57 (2016) 496 [1604.00014]. 
[3] M. Schumann, Direct Detection of WIMP Dark Matter: Concepts and Status, J. Phys. G46 (2019) 103003 [1903.03026].

[4] A. Boveia and C. Doglioni, Dark Matter Searches at Colliders, Ann. Rev. Nucl. Part. Sci. 68 (2018) 429 [1810 . 12238].

[5] J. Beacham et al., Physics Beyond Colliders at CERN: Beyond the Standard Model Working Group Report, J. Phys. G 47 (2020) 010501 [1901.09966].

[6] R. K. Ellis et al., Physics Briefing Book: Input for the European Strategy for Particle Physics Update 2020, 1910.11775.

[7] The European Strategy Group, Deliberation document on the 2020 Update of the European Strategy for Particle Physics, Tech. Rep. CERN-ESU-014, Geneva, 2020. 10.17181/ESU2020Deliberation.

[8] HEP Software Foundation collaboration, A Roadmap for HEP Software and Computing $R$ I\& D for the 2020s, Comput. Softw. Big Sci. 3 (2019) 7 [1712.06982]. 